\title{
Tuning the molecular size of site-specific interferon-polymer conjugate for optimized antitumor efficacy
}

\author{
Guilin Wang ${ }^{\dagger}$, Jin $\mathrm{Hu}^{\dagger}$ and Weiping Gao*
}

\begin{abstract}
The covalent attachment of protein-resistant polymers to therapeutic proteins is a widely used method for extending their in vivo half-lives; however, the effect of molecular weight of polymer on the in vitro and in vivo functions of protein-polymer conjugates has not been well elucidated. Herein we report the effect of molecular weight of poly(oligo(ethylene glycol) methyl ether methacrylate) (POEGMA) on the in vitro and in vivo properties of C-terminal interferon-alpha (IFN)-POEGMA conjugates. Increasing the molecular weight of POEGMA decreased the in vitro activity of IFN- $\alpha$ but increased its thermal stability and in vivo pharmacokinetics. Intriguingly, the in vivo antitumor efficacy of IFN- $a$ was increased by increasing the POEGMA molecular weight from ca. 20 to $60 \mathrm{kDa}$, but was not further increased by increasing the molecular weight of POEGMA from ca. 60 to $100 \mathrm{kDa}$ due to the neutralization of the improved pharmacokinetics and the reduced in vitro activity. This finding offers a new viewpoint on the molecular size rationale for designing next-generation protein-polymer conjugates, which may benefit patients by reducing administration frequency and adverse reactions, and improving therapeutic efficacy.
\end{abstract}

Keywords: protein-polymer conjugate, drug delivery, interferon, tumor therapy

Proteins are of great interest for the treatment of many diseases due to their characteristics of high specificity and activity. However, their widespread use is hampered by their rapid elimination from the circulation, potential immunogenicity and low stability [1-3]. The covalent conjugation of a protein-resistant polymer, typically poly(ethylene glycol) (PEG), to therapeutic proteins, named PEGylation, is a clinically successful strategy to address the above shortcomings. Nevertheless, PEGylation has two major problems: 1) PEG is typically attached to reactive residues of lysine, histidine and cysteine that are randomly distributed on the protein scaffold, so that the conjugate stoichiometry and the conjugation site are out of control and thus results in a heterogeneous product composed of positional isomers, and with reduced biological activity $[4,5] ; 2)$ the yield for PEGylation is generally low (usually $<10 \%$ ) due to the reaction between two large macromolecules, so that the molecular weight of PEG applied in industry is limited to be $40 \mathrm{kDa}$ in consideration of the yield [6]. For instance, recombinant human interferon-alpha (IFN- $\alpha$ ), a potent inhibitor of viral replication and tumor cell growth, has been clinically used for the treatment of viral diseases and cancers, but it suffers from poor pharmacokinetics. To improve its pharmacokinetics, IFN- $\alpha$ is usually conjugated with $40 \mathrm{kDa}$ branched PEG (PEGASYS) or $20 \mathrm{kDa}$ linear PEG (PEGINTRON), but its activity is significantly reduced to $7 \%$ for PEGASYS [7] or $28 \%$ for PEGINTRON [8]. It is anticipated that the activity of protein-polymer conjugate can be well remained by site-specific conjugation at the sites that are distant from the protein's receptor interaction site, such as the $\mathrm{C}$ terminus of IFN- $\alpha$ [9-11].

To date, the effect of molecular size of PEGylated proteins on the in vitro activity and in vivo pharmacokinetics has well been studied, in which increasing the PEG molecular weight decreased the in vitro activity but improved the in vivo pharmacokinetics [10-17]. Nevertheless, the polymer molecular weight effect on the in vivo therapeutic potential has rarely been reported in the literature $[10,15,17]$,

\footnotetext{
Department of Biomedical Engineering, School of Medicine, Tsinghua University, Beijing 100084, China

$\dagger$ These authors contributed equally to this work.

* Corresponding author (email: gaoweiping@tsinghua.edu.cn)
} 
and the preliminary conclusion is that increasing the PEG molecular weight enhanced the in vivo therapeutic efficacy. Unfortunately, the molecular weight of PEG was limited up to $40 \mathrm{kDa}$ in these studies, so that the effect of higher molecular weight of PEG on the function of protein, particularly the in vivo therapeutic potential, has not been elucidated. Additionally, in most of these studies, PEG was randomly conjugated to a protein to yield a mixture of positional isomers, each with distinct in vitro activities, in vivo pharmacokinetic and therapeutic profiles, which further complicated and thus hampered these studies. Hence it is of great interest to synthesize site-specific protein-polymer conjugates with different high molecular weights of polymer to systemically study the effect of molecular size of polymer on the in vitro and in vivo properties of protein.

To this end, herein we report the effect of molecular weight of a PEG-like polymer, poly(oliga(ethylene glycol) methyl ether methacrylate) (POEGMA) on the in vitro activity and stability, in vivo pharmacokinetics and antitumor efficacy of C-terminal IFN- $\alpha$ conjugates of POEGMA. Most recently, we have demonstrated site-specific in situ growth (SIG) of a C-terminal IFN- $\alpha$ conjugate of POEGMA that outperforms PEGASYS in antitumor efficacy [18]. Inspired by this work, we chose to synthesize C-terminal IFN-a conjugates of POEGMA with three high molecular weights of ca. 20, 60 and $100 \mathrm{kDa}$ by using the SIG method that has recently been developed by us [18-22]. We found that increasing the molecular weight of POEGMA decreased the in vitro activity of IFN- $\alpha$ but increased the in vitro stability of IFN- $\alpha$. In a mouse model, the pharmacokinetics of IFN- $\alpha$ was enhanced by increasing the molecular weight of POEGMA. Interestingly, in a tumor-bearing mouse model, the antitumor efficacy of IFN- $\alpha$ was improved by increasing the POEGMA molecular weight from 20 to $60 \mathrm{kDa}$, but was not further enhanced by increasing the POEGMA molecular weight from 60 to $100 \mathrm{kDa}$. To our knowledge, this finding has not been reported in the literature yet.

To synthesize C-terminal IFN-POEGMA conjugates, a triglycine-functionalized initiator for atom transfer radical polymerization (ATRP) was solely attached to the C-terminus of recombinant IFN- $\alpha$ by sortase A mediated ligation to yield a C-terminal IFN- $a$-initiator conjugate (IFN-Br) [18]. Subsequently, in situ ATRP of OEGMA from IFN-Br was performed to yield C-terminal IFN-POEGMA conjugates (Fig. 1a). Based on our previous work in which a C-terminal IFN-POEGMA conjugate with the POEGMA molecular weight of $66.2 \mathrm{kDa}$ outperforms PEGASYS in antitumor efficacy [18], we chose to synthesize three
C-terminal IFN-POEGMA conjugates with POEGMA molecular weights that were below, near and above 66.2 $\mathrm{kDa}$. The molecular weight of POEGMA was controlled by adjusting the feeding molar ratio of OEGMA to IFN-Br. After these in situ ATRP reactions, the ATRP solutions were directly analyzed by gel permeation chromatography (GPC) (Fig. 1b). New GPC peaks corresponding to these IFN-POEGMA conjugates appeared at lower elution times of $19.5,21.1$ and $21.9 \mathrm{~min}$ than that $(30.3 \mathrm{~min})$ for IFN-Br while the GPC peak intensity of IFN-Br decreased, suggesting that the successful in situ growth of the IFN-POEGMA conjugates with different molecular weights, as expected. This result was confirmed by sodium dodecyl sulfate polyacrylamide gel electrophoresis (SDS-PAGE) (Fig. S1, in Supplementary information), which revealed the appearance of new larger molecular weight bands corresponding to the IFN-POEGMA conjugates than that of the band for IFN-Br and simultaneously the decrease of the intensity of the band for IFN-Br. The IFN-POEGMA conjugates were purified by anion exchange chromatography (AEX). GPC traces showed the single peaks for the IFN-POEGMA conjugates without the peak for unreacted IFN-Br (Fig. 1c), demonstrating the successful purification of the IFN-POEGMA conjugates by AEX. This result was also confirmed by SDS-PAGE (Fig. S2), which displayed the bands for the IFN-POEGMA conjugates and the disappearance of the band for the unreacted IFN-Br.

To determine the molecular weights of POEGMA in the IFN-POEGMA conjugates, the IFN part of the conjugates was selectively digested with proteinase $\mathrm{K}$ and the remained POEGMA chains were then analyzed by GPC (Fig. S3). The POEGMA molecular weight parameters and the overall yields of the three IFN-POEGMA conjugates were summarized in Table 1. Based on the molecular weights of POEGMA, herein we name the three IFN-POEGMA conjugates as IFN-POEGMA 20, 60 and $100 \mathrm{kDa}$, respectively. Interestingly, the overall yield of IFN-POEGMA increased with the molecular weight of POEGMA, due to the less loss for IFN-POEGMA with the higher molecular weight of POEGMA during the conjugate purification process. The hydrodynamic radius $\left(R_{\mathrm{h}}\right)$ values of the conjugates determined by dynamic light scattering (DLS) were 5.9, 10.3 and $15.2 \mathrm{~nm}$, respectively, which were 2.7-, 4.8- and 6.9-fold larger than that $(2.2 \mathrm{~nm})$ of IFN- $\alpha$ (Fig. $1 \mathrm{~d})$. The secondary structural conformations of the conjugates were studied by circular dichroism (CD) (Fig. 1e). The CD spectra of them showed a similar doublet at $208 / 222 \mathrm{~nm}$ that matched with that of IFN- $\alpha$, suggesting that the in situ growth of POEGMA with different molecular weights from IFN did 
a

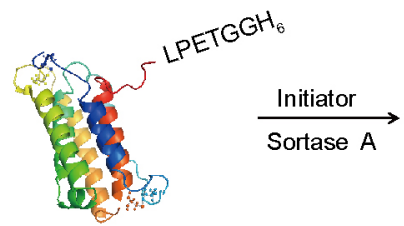

IFN - LPETGGH

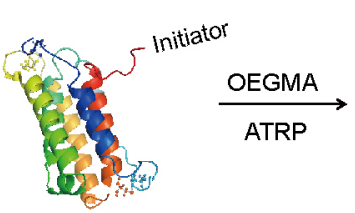

IFN-Br

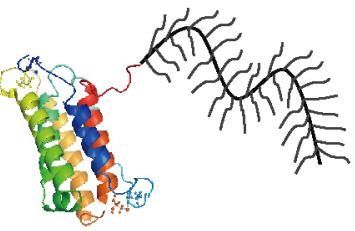

IFN -POEGMA

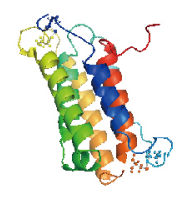

IFN - $\alpha$<smiles>CC(C)(Br)C(=O)OCCNC(=O)CNC(=O)CNC(=O)CN</smiles>

Initiator<smiles>COC(C)(C)CCOC(=O)C12CCC(CC(C)(F)F)(C1)C2</smiles>

POEGMA
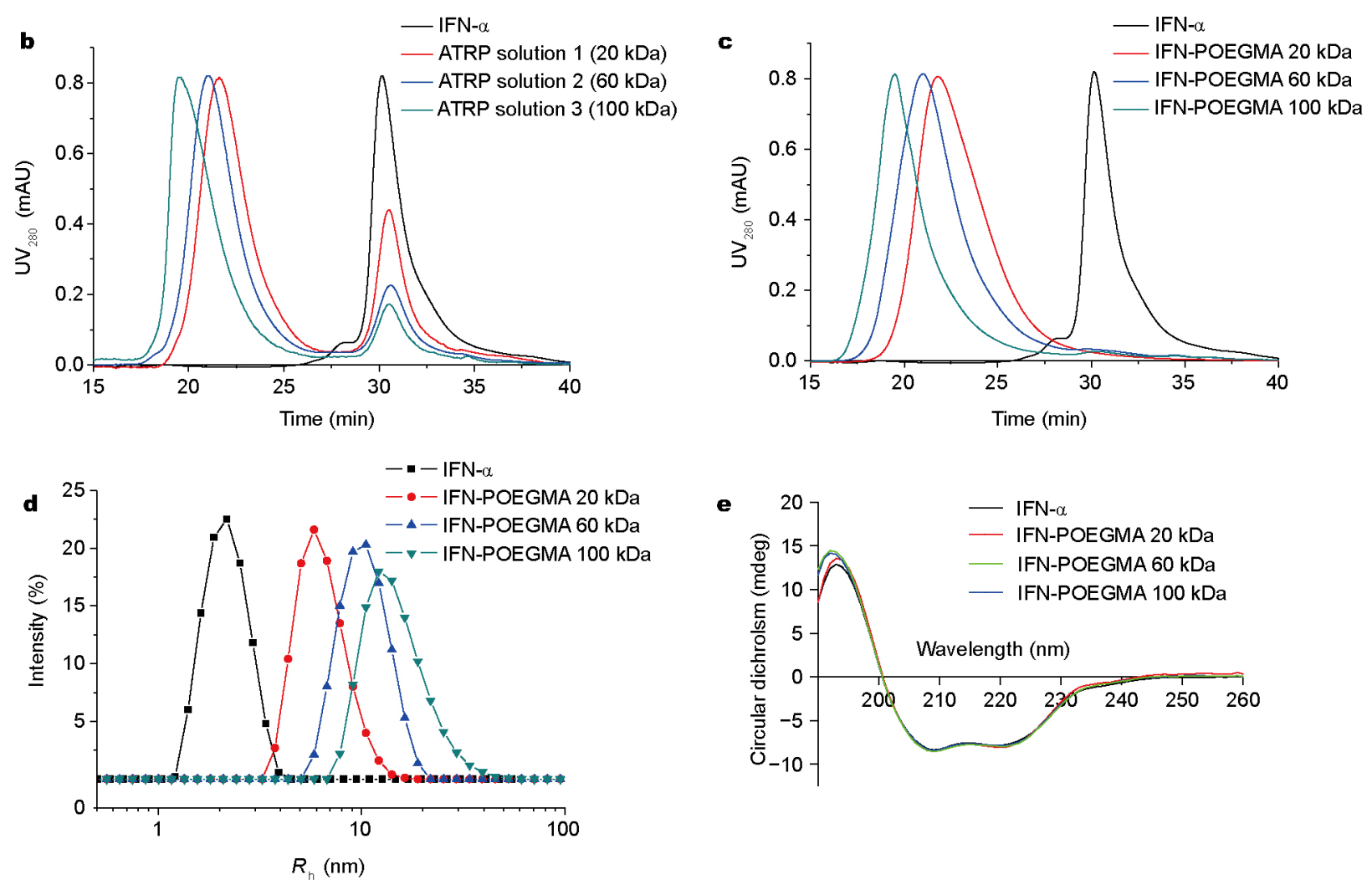

Figure 1 Synthesis and physicochemical characterization of IFN-POEGMA. (a) Synthetic route of C-terminal IFN-POEGMA conjugates. ATRP initiator was site-specifically attached to the C-terminus of IFN- $a$ by sortase A mediated ligation, followed by in situ ATRP of OEGMA from IFN-Br to yield IFN-POEGMA. (b) GPC traces of the ATRP reaction mixtures. (c) GPC traces of the purified IFN-POEGMA conjugates. (d) DLS analyses of the IFN-POEGMA conjugates, where $R_{\mathrm{h}}$ represents hydrodynamic radius. (e) CD analyses of the IFN-POEGMA conjugates.

Table 1 The molecular weights, polydispersities and yields of the IFN-POEGMA conjugates

\begin{tabular}{cccc}
\hline Sample & Number-average molecular weight $\left(M_{\mathrm{n}}\right)(\mathrm{kDa})$ & Molar mass dispersity & Yield $(\%)$ \\
\hline IFN-POEGMA $20 \mathrm{kDa}$ & 23.6 & 1.29 & 38.4 \\
IFN-POEGMA $60 \mathrm{kDa}$ & 64.3 & 1.35 & 63.3 \\
IFN-POEGMA $100 \mathrm{kDa}$ & 104.7 & 1.32 & 79.5 \\
\hline
\end{tabular}


not perturb the secondary structure of IFN.

IFN- $\alpha$ is a highly pleiotropic cytokine, which is widely used to treat a variety of viral diseases and cancers [18,23-25]. We investigated the antiproliferative activity of the conjugates using Daudi B cells (Fig. 2a). The half maximal inhibitory concentrations ( $\mathrm{IC}_{50}$ 's) of IFN-POEGMA 20, 60 and $100 \mathrm{kDa}$ were $17.2,31.2$ and $51.4 \mathrm{pg} \mathrm{mL}^{-1}$, respectively, which indicated $73.1 \%, 40.4 \%$ and $24.5 \%$ relative activities of that of IFN- $\alpha$ (IC50: $12.6 \mathrm{pg} \mathrm{mL}^{-1}$ ), respectively. These data indicated that increasing the molecular weight of POEGMA reduced the activity of IFN (Fig. 2b), which could be attributed to the molecular weight dependence of the physical shielding effect of polymer on the function of protein.

IFN- $\alpha$ is thermally unstable since its antiproliferative activity rapidly decreased to nearly zero after incubation for $1 \mathrm{~h}$ at $50^{\circ} \mathrm{C}$ (Fig. 2c). All of the IFN-POEGMA conjugates showed slower activity decreases than IFN-a, indicating that the POEGMA conjugation could improve the thermal stability of IFN. Additionally, IFN-POEGMA $60 \mathrm{kDa}$ showed a much less activity decrease than IFN-POEGMA $20 \mathrm{kDa}$. Particularly, the relative activity of IFN-POEGMA
$60 \mathrm{kDa}$ was $62 \%$ after incubation for $4 \mathrm{~h}$ at $50^{\circ} \mathrm{C}$, which was 248 -fold higher than that (0.25\%) of IFN-POEGMA 20 $\mathrm{kDa}$. IFN-POEGMA $100 \mathrm{kDa}$ also showed a slower activity decrease than IFN-POEGMA $60 \mathrm{kDa}$. These data indicated that increasing the molecular weight of POEGMA could increase the thermal stability of IFN. This result was further supported by $\mathrm{CD}$ analysis (Fig. 2d). After incubation for $24 \mathrm{~h}$ at $50^{\circ} \mathrm{C}$, all of the conjugates showed higher intensities of the doublet at $208 / 222 \mathrm{~nm}$ than IFN- $\alpha$, indicating the higher $\alpha$-helical structure retention of the conjugates than IFN- $\alpha$. Furthermore, increasing the molecular weight of POEGMA improved the a-helical structure retention of IFN, as indicated by the higher intensity of the doublet of the conjugate with the higher molecular weight of POEGMA. Taken together, these data indicated that increasing the molecular weight of POEGMA could improve the thermal stability of IFN in activity and structure.

Next, we studied the in vivo properties of the IFN-POEGMA conjugates in mouse models. At first, we evaluated the pharmacokinetics of the conjugates by analyzing the plasma samples at various time points after their intravenous injection (Fig. 3a). A two-compartment
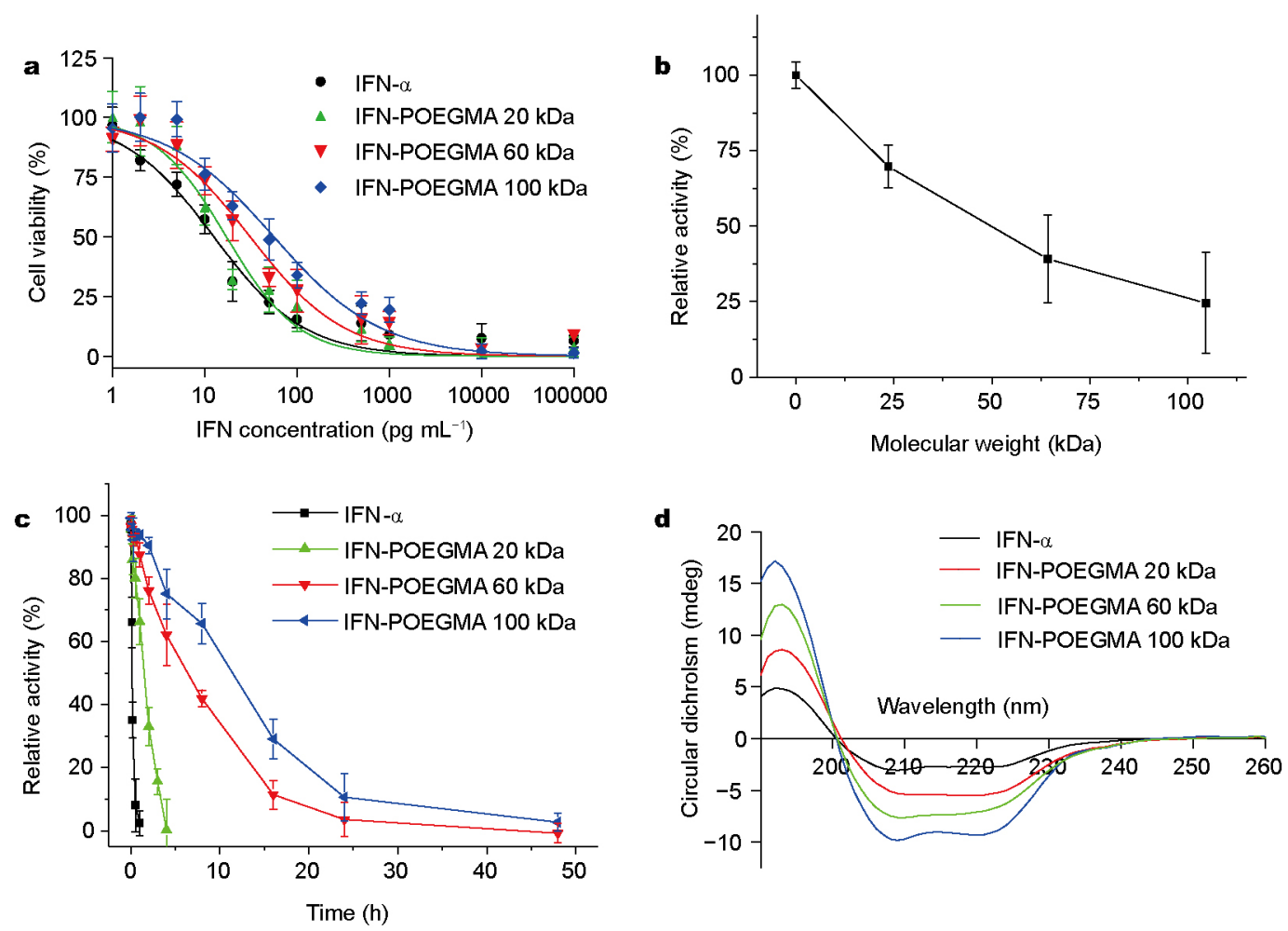

Figure 2 The in vitro anti-proliferative activity and thermal stability of IFN-POEGMA. (a) The in vitro cytotoxicity of IFN-POEGMA. (b) The relative activity of IFN-POEGMA as a function of molecular weight. (c) The thermal stability (in terms of relative activity retention) of IFN-POEGMA after incubation at $50^{\circ} \mathrm{C}$, where the number represents the molecular weight of POEGMA. (d) CD spectra of IFN- $\alpha$ and IFN-POEGMA after incubation for $24 \mathrm{~h}$ at $50^{\circ} \mathrm{C}$. 

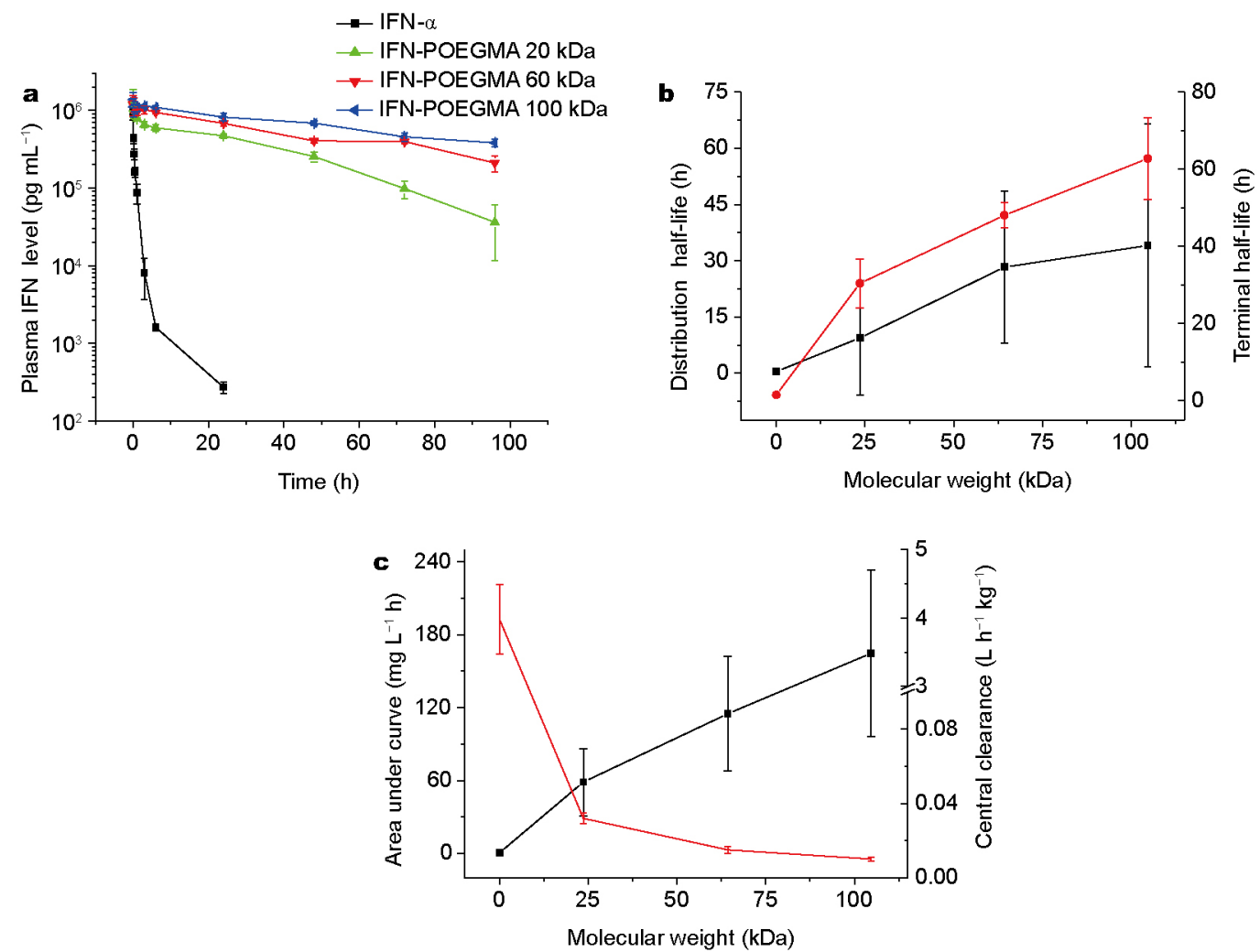

Figure 3 The in vivo pharmacokinetic profiles of IFN-POEGMA. (a) The plasma IFN concentration as a function of time post injection. ( $P<0.001$ for IFN-POEGMA $v s$. IFN- $\alpha$ ). (b) The distribution and terminal half-life as a function of molecular weight, where the number represents the molecular weight of POEGMA. (c) The area under curve and central clearance as a function of molecular weight, where the number represents the molecular weight of POEGMA. Data are shown as mean $\pm \mathrm{SD}(n=3)$.

model was used to fit the data and the representative pharmacokinetic parameters of the conjugates and IFN- $\alpha$ were summarized in Table S1. IFN- $\alpha$ was rapidly cleared from plasma with a high central clearance rate of $3.98 \mathrm{Lh}^{-1} \mathrm{~kg}^{-1}$, and exhibited a short distribution half-life $\left(t_{1 / 2 \alpha}=0.44 \mathrm{~h}\right)$ and terminal half-life $\left(t_{1 / 2 \beta}=1.52 \mathrm{~h}\right)$. This rapid clearance is because IFN- $\alpha$ has a small size of $2.2 \mathrm{~nm}$ in $R_{\mathrm{h}}$ that is much smaller than the effective size cutoff of kidney (ca. $5 \mathrm{~nm}$ in $R_{\mathrm{h}}$ [11]). In contrast, the distribution half-lives $\left(t_{1 / 2 \alpha}=9.4,28.3\right.$ and $\left.34.1 \mathrm{~h}\right)$ of the conjugates were increased by $21.4,64.4$ and 77.4 times as compared to that of IFN- $\alpha$, respectively, and the terminal half-lives $\left(t_{1 / 2 \beta}=30.4,48.1\right.$ and $\left.62.8 \mathrm{~h}\right)$ of the conjugates were prolonged by $20.0,31.6$ and 41.3 times relative to IFN- $\alpha$, respectively (Fig. $3 b$ ). The central clearance rate of the IFN-POEGMA 20, 60 and $100 \mathrm{kDa}$ conjugates decreased to $0.03,0.02$ and $0.01 \mathrm{Lh}^{-1} \mathrm{~kg}^{-1}$, respectively (Fig. $3 c)$. This reduced clearance could be attributed to the increased hydrodynamic sizes $(5.9,10.3$ and $15.2 \mathrm{~nm}$ in $R_{\mathrm{h}}$ ) of the IFN-POEGMA conjugates that were well above the effective size cutoff of kidney. The differences in pharmacokinetics resulted in 85.1-, 166.6- and 238.6-fold increases in the area under the curve (AUC) of the conjugates $\left(58.7,114.9\right.$ and $164.6 \mathrm{mg} \mathrm{L}^{-1} \mathrm{~h}$ ) relative to that of IFN- $\alpha\left(0.69 \mathrm{mg} \mathrm{L}^{-1} \mathrm{~h}\right)$, respectively (Fig. 3c), indicating that increasing the POEGMA molecular weight improved the plasma exposure of IFN. Taken together, these data showed that increasing the molecular weight of POEGMA could enhance the pharmacokinetics of IFN- $\alpha$ due to the increased hydrodynamic sizes of the conjugates with the increased molecular weights of POEGMA.

To investigate the in vivo antitumor efficacy of the IFN-POEGMA conjugates (Fig. 4), we treated nude mice bearing ovarian tumors of $20 \mathrm{~mm}^{3}$ with the conjugates ( $20 \mu \mathrm{g}$ IFN Equiv/mouse, weekly) until the tumors in all the control groups grew up to $500 \mathrm{~mm}^{3}$ (Fig. 4a). IFN- $a$ did not show obvious inhibition on tumor growth relative to the negative control of saline. In contrast, IFN-POEGMA $20 \mathrm{kDa}$ significantly inhibited tumor growth. Interestingly, IFN-POEGMA $60 \mathrm{kDa}$ showed the same antitumor efficacy as IFN-POEGMA $100 \mathrm{kDa}$, as indicated by their complete inhibition on tumor growth. These results were 

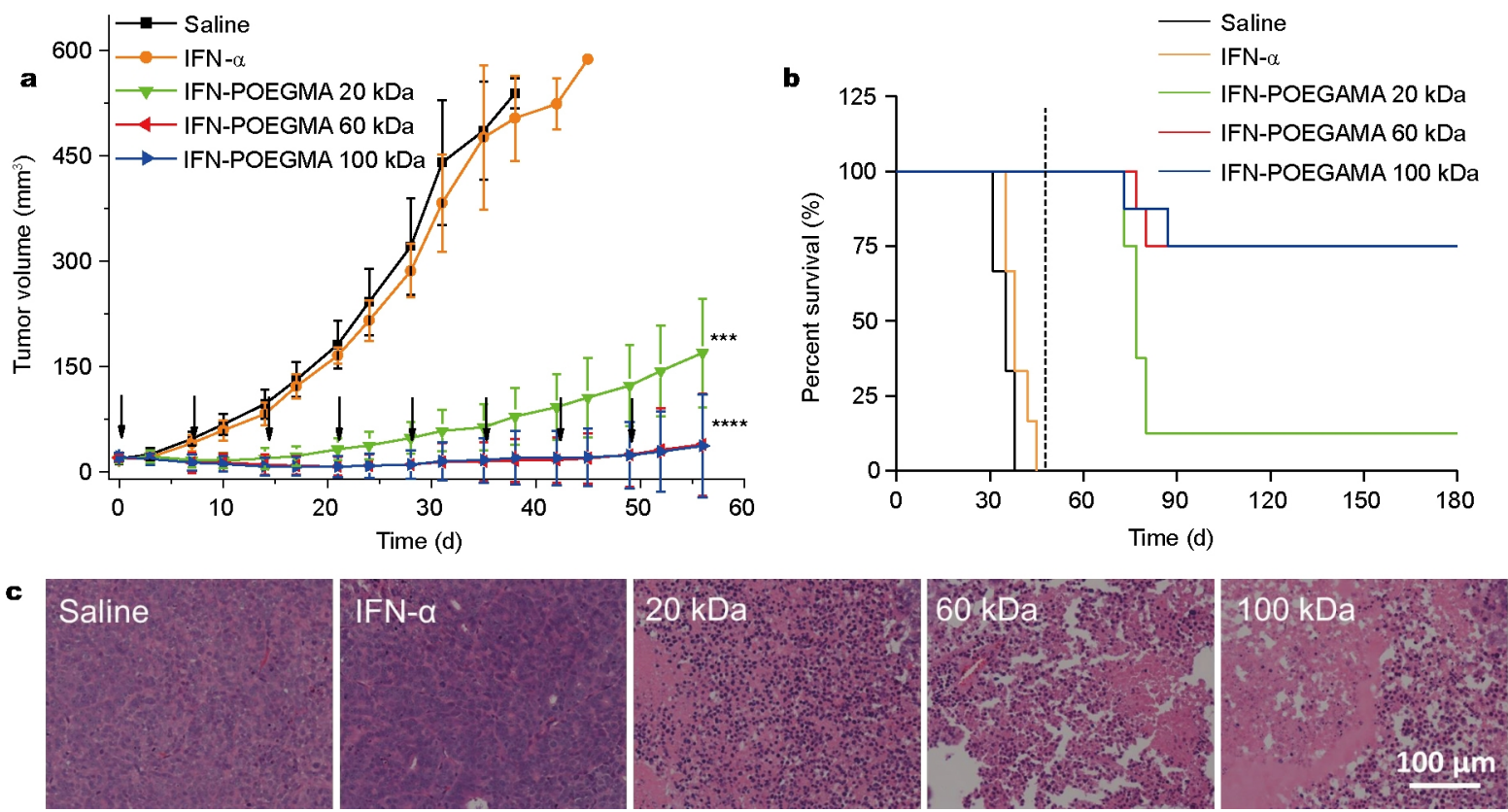

Figure 4 The in vivo antitumor efficacy of IFN-POEGMA. (a) Inhibition of tumor growth after weekly treatment, as indicated by the arrows. $\left({ }^{* *} P\right.$ $<0.001$ for IFN-POEGMA $20 \mathrm{kDa} v s$. IFN- $\alpha,{ }^{* * * *} P<0.0001$ for IFN-POEGMA 60 and $100 \mathrm{kDa} v s$. IFN- $\alpha$ ). (b) Survival curves of mice. The vertical dashed line indicates the ending date of treatment. ( ${ }^{* *} P<0.001$ for IFN-POEGMA $v s$. IFN- $\alpha$ ). (c) Histological evaluation of tumors. Data are shown as mean $\pm \mathrm{SD}(n=5-8)$.

related to animal survival after treatment (Fig. 4b). The median survival time (42 days) for the mice treated with IFN- $\alpha$ was almost similar to that (38 days) for the mice treated with saline. In contrast, the median survival time (77 days) for the mice treated with IFN-POEGMA $20 \mathrm{kDa}$ was 1.83 -fold longer than that (42 days) for the mice treated with IFN- $\alpha$. Particularly, the median survival times for the mice treated with IFN-POEGMA 60 and $100 \mathrm{kDa}$ were not available as $75 \%$ mice in each group were tumor-free. The antitumor efficacy of the conjugates was verified by hematoxylin and eosin (H\&E) staining of the tumors (Fig. 4c). The tumors of the mice treated with saline and IFN- $\alpha$ showed closely-packed tumor cells with intact structure, while the tumors of the mice treated with IFN-POEGMA $20 \mathrm{kDa}$ showed vacuolization, indicating that IFN-POEGMA $20 \mathrm{kDa}$ caused damage to tumor cells. As expected, IFN-POEGMA 60 and $100 \mathrm{kDa}$ induced severe damage to tumor cells, as indicated by the extensive vacuolization. Taken together, IFN-POEGMA $60 \mathrm{kDa}$ showed better antitumor efficacy than IFN-POEGMA $20 \mathrm{kDa}$, while IFN-POEGMA 100 $\mathrm{kDa}$ showed similar antitumor efficacy to IFN-POEGMA $60 \mathrm{kDa}$. Considering that the AUC of IFN-POEGMA 100 $\mathrm{kDa}$ was 1.4-fold higher than that of IFN-POEGMA 60 $\mathrm{kDa}\left(164.6\right.$ vs. $\left.114.9 \mathrm{mg} \mathrm{L}^{-1} \mathrm{~h}\right)$, whereas the in vitro antiproliferative activity (24.5\% retention) of IFN-POEGMA
$100 \mathrm{kDa}$ was 1.6 -fold lower than that (40.4\% retention) of IFN-POEGMA $100 \mathrm{kDa}$, we think that the improved in vivo pharmacokinetics was offset by the reduced in vitro activity, which resulted in the similar antitumor efficacy. It should be pointed out that while the ovarian tumor model successfully demonstrated the in vivo antitumor effect of IFN- $\alpha$ and its polymer conjugates, no inhibitory effect was observed for ovarian carcinoma cells (OVCAR-3) in in vivo antiproliferative test. This indicates a complicated mechanism for the in vitro and in vivo antitumor effect, and more investigations are needed in order to better understand how the IFN- $\alpha$ inhibits tumor growth.

We also examined the biosafety of the conjugates. No loss of body weight was found for the mice treated with the conjugates and IFN- $\alpha$ (Fig. S4). No apparent histological changes in major organs of heart, liver and kidney were observed after H\&E staining of the organs of the mice treated with the conjugates, IFN- $\alpha$ and saline (Fig. S5). There were no significant changes in blood biochemistry parameters related to heart, liver and kidney function markers for the mice treated with the conjugates, IFN- $\alpha$ and saline (Fig. S6). The major hematological indicators such as red and white blood cells, platelets and hemoglobin for the mice treated with the conjugates and IFN- $\alpha$ appeared to be normal relative to those for the mice treated with saline (Fig. S7). These results indicated that increasing the molecular 
weight of POEGMA did not induce the systemic toxicity of IFN.

In conclusion, we report the effect of molecular weight of POEGMA on the in vitro and in vivo properties of C-terminal IFN-POEGMA conjugates. These conjugates with defined molecular weights of POEGMA were synthesized by the SIG method developed by us, which made it possible to systemically study the polymer molecular weight effect on the in vitro and in vivo properties of protein-polymer conjugates. This study leads to three major findings that are important for the design of advanced protein-polymer conjugates for disease treatment: (i) increasing the polymer molecular weight decreases the in vitro activity of the protein but improves the thermal stability of the protein, due to the molecular weight dependence of the physical shielding of polymer on the function of protein; (ii) increasing the polymer molecular weight enhances the in vivo pharmacokinetics of the protein because of the enlarged hydrodynamic size; (iii) increasing the polymer molecular weight does not always enhance the therapeutic efficacy of the protein as the improved in vivo pharmacokinetics may be offset by the decreased in vitro activity, which has not been reported in the literature. We believe that this new finding is of considerable interest as a novel molecular size rationale for the design of a number of next-generation protein/peptide-polymer conjugates for the treatment of various diseases.

Received 10 May 2017; accepted 22 May 2017; published online 2 June 2017

1 Elliott S, Lorenzini T, Asher S, et al. Enhancement of therapeutic protein in vivo activities through glycoengineering. Nat Biotech, 2003, 21: 414-421

2 Zhao W, Liu F, Chen Y, et al. Synthesis of well-defined protein-polymer conjugates for biomedicine. Polymer, 2015, 66: A1-A10

3 Leader B, Baca QJ, Golan DE. Protein therapeutics: a summary and pharmacological classification. Nat Rev Drug Discov, 2008, 7: 21-39

4 Veronese FM. Peptide and protein PEGylation. Biomaterials, 2001, 22: $405-417$

5 Seely JE, Richey CW. Use of ion-exchange chromatography and hydrophobic interaction chromatography in the preparation and recovery of polyethylene glycol-linked proteins. J Chromatography A, 2001, 908: 235-241

6 Shaunak S, Godwin A, Choi JW, et al. Site-specific PEGylation of native disulfide bonds in therapeutic proteins. Nat Chem Biol, 2006, 2: 312-313

7 Reddy KR, Modi MW, Pedder S. Use of peginterferon alfa-2a (40 $\mathrm{KD})\left(\right.$ Pegasys $\left.^{\circledR}\right)$ for the treatment of hepatitis C. Adv Drug Deliver Rev, 2002, 54: 571-586

8 Wang YS, Youngster S, Grace M, et al. Structural and biological characterization of pegylated recombinant interferon alpha- $2 \mathrm{~b}$ and its therapeutic implications. Adv Drug Deliver Rev, 2002, 54:
$547-570$

9 Popp MW, Dougan SK, Chuang TY, et al. Sortase-catalyzed transformations that improve the properties of cytokines. Proc Natl Acad Sci USA, 2011, 108: 3169-3174

10 Grace MJ, Lee S, Bradshaw S, et al. Site of PEGylation and polyethylene glycol molecule size attenuate interferon- antiviral and antiproliferative activities through the JAK/STAT signaling pathway. J Biol Chem, 2005, 280: 6327-6336

11 Caliceti P. Pharmacokinetic and biodistribution properties of poly(ethylene glycol)-protein conjugates. Adv Drug Deliver Rev, 2003, 55: 1261-1277

12 DeNardo SJ, Yao Z, Lam KS, et al. Effect of molecular size of PEGylated peptide on the pharmacokinetics and tumor targeting in lymphoma-bearing mice. Clin Cancer Res, 2003, 9: 3854s-3864s

13 Hee Na D, Seok Youn Y, Bok Lee I, et al. Effect of molecular size of PEGylated recombinant human epidermal growth factor on the biological activity and stability in rat wound tissue. Pharma Dev Tech, 2006, 11: 513-519

14 Zhai Y, Zhao Y, Lei J, et al. Enhanced circulation half-life of site-specific PEGylated rhG-CSF: optimization of PEG molecular weight. J Biotech, 2009, 142: 259-266

15 Bowen S, Tare N, Inoue $\mathrm{T}$, et al. Relationship between molecular mass and duration of activity of polyethylene glycol conjugated granulocyte colony-stimulating factor mutein. Exp Hematology, 1999, 27: 425-432

16 Yang K, Basu A, Wang M, et al. Tailoring structure-function and pharmacokinetic properties of single-chain Fv proteins by site-specific PEGylation. Protein Eng Des Selection, 2003, 16: 761-770

17 Fam CM, Eisenberg SP, Carlson SJ, et al. PEGylation improves the pharmacokinetic properties and ability of interferon gamma to inhibit growth of a human tumor xenograft in athymic mice. J Interferon Cytokine Res, 2014, 34: 759-768

$18 \mathrm{Hu}$ J, Wang G, Zhao W, et al. Site-specific in situ growth of an interferon-polymer conjugate that outperforms PEGASYS in cancer therapy. Biomaterials, 2016, 96: 84-92

19 Gao W, Liu W, Mackay JA, et al. In situ growth of a stoichiometric PEG-like conjugate at a protein's N-terminus with significantly improved pharmacokinetics. Proc Natl Acad Sci USA, 2009, 106: 15231-15236

20 Gao W, Liu W, Christensen T, et al. In situ growth of a PEG-like polymer from the $\mathrm{C}$ terminus of an intein fusion protein improves pharmacokinetics and tumor accumulation. Proc Natl Acad Sci USA, 2010, 107: 16432-16437

21 Hu J, Zhao W, Gao Y, et al. Site-specific in situ growth of a cyclized protein-polymer conjugate with improved stability and tumor retention. Biomaterials, 2015, 47: 13-19

22 Zhang L, Zhao W, Liu X, et al. Site-selective in situ growth of fluorescent polymer-antibody conjugates with enhanced antigen detection by signal amplification. Biomaterials, 2015, 64: 2-9

23 Hu J, Wang G, Liu X, et al. Enhancing pharmacokinetics, tumor accumulation, and antitumor efficacy by elastin-like polypeptide fusion of interferon alpha. Adv Mater, 2015, 27: 7320-7324

24 Pfeffer LM, Dinarello CA, Herberman RB, et al. Biological properties of recombinant $\alpha$-interferons: 40th anniversary of the discovery of interferons. Cancer Res, 1998, 58: 2489-2499

25 Bailon P, Palleroni A, Schaffer CA, et al. Rational design of a potent, long-lasting form of interferon: a $40 \mathrm{kDa}$ branched polyethylene glycol-conjugated interferon $\alpha-2 a$ for the treatment of hepatitis $C$. Bioconjugate Chem, 2001, 12: 195-202

Acknowledgments This study was financially supported by Grants from the National Natural Science Foundation of China (21274043 and 


\section{6).}

Author contributions Gao W and Wang G designed the project; Wang $\mathrm{G}$ and $\mathrm{Hu}$ J synsthesized and purified the samples and carried out all the analyses; $\mathrm{Hu} \mathrm{J}$ completed the H\&E staining, biochemistry and hematological investigations; Wang $\mathrm{G}$ and $\mathrm{Hu} J$ wrote the paper with support from Gao W. All authors contributed to the general discussion.

Conflict of interest The authors declare that they have no conflict of interest.
Supplementary information Materials, full experimental details including synthesis, physicochemical characterization, in vitro cytotoxicity and thermal stability, pharmacokinetics, in vivo antitumor efficacy and systemic toxicity. Supporting data including SDS-PAGE analysis, pharmacokinetic parameters, the change of mouse body weight post administration, H\&E staining, clinical biochemistry and hematological parameters for mice, are available in the online version of the paper.
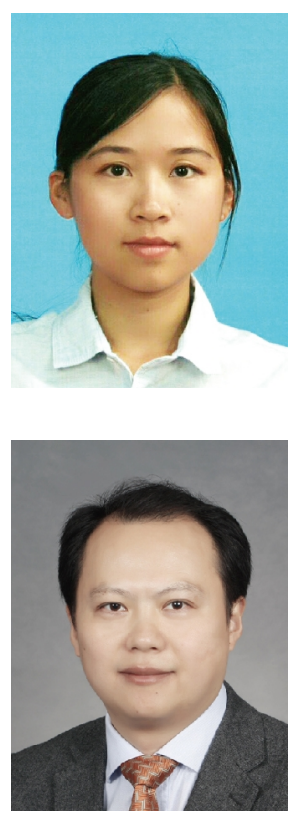

Jin Hu received her bachelor's degree in biomedical engineering from Nanchang University, China, in 2012. She is currently a PhD candidate at the Department of Biomedical Engineering, School of Medicine, Tsinghua University. Her research is focused on the synthesis of site-specific polymer-protein conjugates and their pharmacological properties.
Weiping Gao is a professor at the Department of Biomedical Engineering, School of Medicine, Tsinghua University. He received his PhD degree from Peking University in 2004. From 2005 to 2007, he worked as a researcher at Kyoto University, Japan, and from 2007 to 2011, he was a postdoctoral associate at the Department of Biomedical Engineering and Center for Biologically Inspired Materials and Material Systems, Duke University, USA. His research interests include the synthesis of polymer-biomolecule conjugates and the application of nanotechnology in biomedicine.

\section{调节位点特异性干扰素-聚合物偶联物的分子量以优化抗癌作用}

王贵林 ${ }^{\dagger}$, 胡瑾 ${ }^{\dagger}$ 高卫平*

摘要 治疗性蛋白质共价结合蛋白质抗性聚合物是被广泛使用的延长其体内半衰期的方法. 然而, 聚合物的分子量对蛋白质 - 聚合物偶 联物体外和体内性能的影响尚未得到很好的阐明. 本文报道了聚(寡聚乙二醇甲基丙烯酸酯) (POEGMA)的分子量对C末端修饰干扰素- $\alpha$ (IFN)-POEGMA偶联物的体外和体内性能的影响. 增加POEGMA的分子量会降低IFN- $\alpha$ 的体外活性, 但改善了其热稳定性和体内药代动力 学. IFN- $\alpha$ 的体内抗肿瘤功效随着POEGMA分子量从 20 增加至 $60 \mathrm{kDa}$ 而增强, 但进一步提高POEGMA的分子量到 $100 \mathrm{kDa}$, 由于其生物活性 的降低中和了药代动力学的改善作用, 并不能进一步增强其体内抗肿瘤功效. 该发现为蛋白质 - 聚合物偶联物分子尺寸效应提供了新的佐 证, 可为设计下一代蛋白质-聚合物偶联物提供参考, 通过减少给药频率和不良反应并改善治疗效果而使患者受益. 